\title{
The HIV/AIDS Situation in Europe : Available Data and Prospects for Public Health Surveillance
}

\author{
Jean Baptiste Brunet
}

\begin{abstract}
The European region counts some $\mathbf{8 5 0}$ milions inhabitants living in $\mathbf{5 0}$ different countries, and the analysis of the epidemic situation reveals complex patterns of pictures and dynamics. Such analysis are facilitated by the fact that a common information system has been developped in the European region since 1984 . 44 out of the 50 countries are participating in that system, by providing standardized AIDS/HIV surveillance data to the European Centre.

J Epidemiol,1996; 6 : S129-S131.
\end{abstract}

AIDS, Public health, Europe

\section{RESULTS}

By 31 December 1995, a cumulative total of 160982 AIDS cases including 154866 adult/adolescent and 6060 paediatric cases had been reported in the region. A total of 26139 AIDS cases were reported in 1995, an increase of less than $1 \%$ compared to $1994^{1}$. Over the past 2 to 3 years, AIDS incidence appears to have stabilized in the northern part of western Europe, mostly due to a stable or declining incidence of cases among homosexual males. It is still increasing in countries of southern Europe where AIDS incidence is primarily related to injecting drug use. In central and eastern Europe (with the exception of Romania), the HIV/AIDS epidemic is more recent and AIDS incidence much lower than in western Europe.

Before 1990 most AIDS cases were diagnosed in male homo/bisexuals, however, since 1990 , injecting drug users (IDUs) account for the highest proportion of yearly diagnosed cases in the region (43\% of adult/adolescent cases in 1995). Between 1994 and 1995, the number of cases diagnosed in male homo/bisexuals decreased by $9 \%$ and that in IDUs by $3 \%$ while the number of cases in heterosexually infected patients increased by $4 \%$. The proportion of female cases rose from $11 \%$ in 1986 to $20 \%$ in 1995 . Most women diagnosed with AIDS in 1995 , were IDUs $(46 \%)$ or had been heterosexually- infected $(40 \%)$, often by an IDU sex partner (33\% of heterosexually-infected women). In most countries, the vast majority of children have been infected by mother-to-child transmission. However, in Romania and Russia, most pediatric cases have been infected through nosocomial transmission of HIV2).

\section{HIV INCIDENCE AND PREVALENCE}

In western countries, reconstruction of past trends of HIV incidence through back calculation models usually shows incidence peaks in the mid $80 \mathrm{~s}$, with large differences in intensity according to countries and transmission groups. The same method shows a low but steady increase of HIV prevalence among heterosexual populations. Trends for I.V.D.U. appears more variable and complex. Birth cohort analysis of AIDS cases suggest that HIV transmission through injecting drug use among young adults decreased in the early 90 s in France, Italy, and Switzerland, but increased over the same period in Spain and Portugal ${ }^{33}$.

Back calculations performed in western Europe in 1996 provided an estimate of around 450000 adults living with HIV at the end of 1993, with an annual incidence of around 40000 since the beginning of the $90 \mathrm{~s}^{4}$. In many countries of eastern Europe estimates of HIV prevalence are more uncertain. Until recently, HIV reporting systems associated with systematic

European Centre for the Epidemiological Monitoring of AIDS, Hospital National de Saint Maurice, Saint Maurice, France. Address for correspondence : Jean Baptiste Brunet, European Centre for the Epidemiological Monitoring of AIDS, Hospital National de Saint Maurice, 14 rue du Val d'Osne 94410 Saint Maurice, France. 
testing of large segments of the general population had not identified increasing trends of HIV incidence. The possibility of having recent rapid increases in HIV incidence in some of these countries makes any estimate of prevalence extremely hazardous.

Among pregnant women, the highest prevalence rates are observed in the regions of Paris, Rome, Milan, London, Madrid, Barcelona, and Amsterdam (between 1 and 4 per thousand) In northern and eastern Europe, prevalence rates among pregnant women are much lower (between 0 . and 0.1 per thousand). In 1995, despite systematic antenatal screening, no HIV positive pregnant women has been found in Bulgaria, Lithuania, Moldova, Norway, and the Slovak Republic ${ }^{5}$.

Among 87,640 patients tested in sexually transmitted diseases (STD)clinics from 17 countries (Czech Republic, Hungary, Norway, Switzerland, and countries of the European Union except Luxembourg and Ireland), 2.8\% were HIV positive. Homo/bisexual men had the highest HIV infection rate in most countries (between 10\% and 50\%) except in Italy, Spain and Switzerland, where the highest rates were found for IDUs. Among the non IDU heterosexual STD patients, HIV rates were usually 10 to 30 times lower than among homo/bisexual men, with rates below $1 \%$ in 11 countries. Higher rates (between 1 and 3\%) were found in France, Germany, Italy, Portugual, Spain and Switzerland ${ }^{(6)}$.

Available results from systematic screening of STD patients in several countries of eastern Europe have shown in the recent past very low rates of HIV infection, as in Russia where 64 HIV positive patients were identified from nearly 6 milion tests done between 1/87 and12/937. However, dramatic ongoing changes in STD incidence in these countries demonstrate a potential for a rapid change in HIV dynamics.

Injecting drug users : in Madrid, percentages of HIV infected IDUs entering detoxification programmes ranged between $74 \%$ to $59 \%$ during 1986 to 1990 . In Italy, on a national level, percentage of HIV infected IDUs was $31 \%$ and $39 \%$ in 1990 and 1991 respectively. Among eastern countries, large outbreaks of HIV infection in IDUs have been observed in the late eighties in the former republic of Yougoslavia, and Poland, where $46 \%$ of IDUs entering detoxification centres in Warsaw in 1993 were reported HIV infected ${ }^{8}$. A dramatic outbreak was also reported recently in Ukrainian cities bordering the Black Sea, where the percentage of HIV infected IDU rose from 1,3$1.7 \%$ to $30-50 \%$ from january to december 1995 (Y. Kobyshcha, personal communication).

\section{BEHAVIOUR CHANGE}

Behavioural surveys carried on in western countries in 19871990 shown that the reported numbers of sexual partners were quite stable over the period, irrespective of the country, while condom use increased markedly, particularly for the most sex- ually active population groups ${ }^{9}$. Among people with casual partners, the percentage of those reporting always using condoms rose from $8 \%$ in 87 to $48 \%$ in 89 in Switzerland, from $9 \%$ to $40 \%$ in the Netherlands. In the U.K., among 18-24 years old, the percentage of those having used a condom on last occasion of having sex rose from $14 \%$ to $31 \%$ between 86 and 89 . Such results are partially supported by trends in condom sales. In Switzerland, main wholesalers have increased their sales from 7.6 milion units in 1986 to 15 milions in 1992. In France, the number of condom sold in pharmacies and supermarkets rose from 38.6 milions to 74.4 milions between 1986 and 1993.

Worrisome information arose recently from the independent states of the former Soviet Union. In 1995, compared to 1994, syphilis incidence rates per 100000 population rose from 81.7 to 172 in Russia (from 169.8 to 320.8 in St Petersbourg), from 72.1 to 147.1 in Belarus, from 116.6 to 173.6 in Moldova, from 32.6 to 123 in Kazakhstan (A. Gromyko, personal communication). These results indicates not only the likelihood of further spread of other STDs (including HIV infection), but also a potential for further dissemination in neighbouring countries. This is already happening in Finland, where 118 new syphilis cases where diagnosed in 1995, compared to 63 in 1994. Close links with the Russian epidemic were identified, through the increase of Finnish business/pleasure tourism in the St Petersburg area, and migration from Russia to Finland ${ }^{10}$.

\section{DISCUSSION}

European countries are now facing different situations, potentially explosive or exploding epidemics, endemic situations with high or low HIV prevalence levels, and even decreasing incidence rates. Transmission of HIV through I.V. drug use is responsible for the majority of cases in some of the western countries which have the highest incidence of AIDS, (Spain, Italy) and is strongly associated with cases appearing among heterosexuals and children in the same countries. In eastern countries, the more serious HIV outbreaks reported until now (Poland, Ukraine) are also associated with I.V.drug use.

Although the relative proportion of homo/bisexual men among AIDS cases has steadily decrease in the past ten years in the region, this is mainly due to a comparatively more rapid progression of cases among drug users and to the low but steady increase of the proportion of heterosexuals cases. When AIDS incidence among gay men is taken in account, it appears only moderately declining or quite stable in most western countries, and still increasing in Greece, Portugal and Norway. In several eastern countries, like in the Baltic States, Slovenia and Hungary, homosexual men count for the vast majority of HIV infections reported so far among males. Moreover, information from the United Kingdom indicates that a reversal of 
the declining trend of homosexual transmission observed in that country in the late eighties may have actually happened since 199011).

The past and present experience about the HIV/AIDS dynamic in the European region indicates that surveillance efforts, as well as prevention programs and harm reduction policies, should be focussed on the populations at greatest risk of HIV infection, particularly in still low prevalence countries which are facing potentially explosive situations. Such efforts should include HIV prevalence surveys in selected sentinel populations, and STD surveillance, but also sharing injection equipment and sexual behaviour surveys which are of primary importance in that respect. Process indicators of prevention efforts, like condoms and injection equipment availability and sales should also be considered as basic elements of HIV/AIDS surveillance programs.

\section{REFERENCES}

1. European Centre for the Epidemiological Monitoring of AIDS. HIV/AIDS surveillance in Europe. Quaterly report no 48, 31 December 1995. St Maurice, 1995 : 7-41.

2. Hersh BC, Popovici F, Apeitrei RC, et al. Acquired immunodeficiency virus in Romania. Lancet 1991 ; 338 : 645-649.

3. Hamers F, Downs AM, Alix J, Brunet JB. AIDS trends among young birth cohorts in western Europe suggest changes in past HIV transmission through drug use. Intl. Conf. Aids 1996; 1 Abstract Mo. C 1547.
4. Downs A, Heisterkamp SH, Hamers F, Brunet JB. Empirical Bayesian back-calculations of the HIV epidemic in the European Union. Intl. Conf. AIDS 1996; 1 Abstract Mo. C 1571.

5. European Centre for the Epidemiological Monitoring of AIDS. HIV Prevalence among pregnant women. Quaterly report no 44, 31 December 1994. St Maurice, 1994 : 41-53.

6. The European Study Group : European Community concerted action on HIV seroprevalence among sexually transmitted disease patients in 18 European sentinel networks. AIDS, $1993 ; 7: 393-400$.

7. Chaika N. Recent data on HIV/AIDS epidemic in eastern Europe. St Petersburg Pasteur Institute Report, 1995 : 7.

8. European Centre for the Epidemiological Monitoring of AIDS. HIV Prevalence among injecting drug users in Europe. Quaterly report no 50, 30 June 1996. St Maurice, 1996 : 42-46.

9. Wellings K. Assessing AIDS/HTV prevention : what do we know in Europe ? Sozial und Praeventivmedizin, $1994 ; 39$ Suppl $1: 14-46$.

10. Hiltunen-Back E, Haikala O, Koskela P, Reunala T. Increase of Syphilis in Finland related with the Russian epidemic. Eurosurveillance, $1996 ; 1: 1-2$.

11. Evans B, Catchpole MA, Hepstonstall J, et al. Sexually transmitted disease and HIV infection among Homosexual males in England and Wales. B.M.J., 1993 ; $306: 426-428$. 